\title{
A comparison of animal personality and coping styles in the red junglefowl
}

J osefina Zidar, Alexandra Balogh, Anna Favati, Per J ensen, Olof Leimar and Hanne Lovlie

The self-archived version of this journal article is available at Linköping University Institutional Repository (DiVA):

http:// urn.kb.se/ resolve?urn=urn:nbn:se:liu:diva-139903

N.B.: When citing this work, cite the original publication.

Zidar, J ., Balogh, A., Favati, A., J ensen, P., Leimar, O., Lovlie, H., (2017), A comparison of animal personality and coping styles in the red junglefowl, Animal Behaviour, 130, 209-220.

https:// doi.org/ 10.1016/j.anbehav.2017.06.024

Original publication available at:

https:// doi.org/ 10.1016/j.anbehav.2017.06.024

Copyright: Elsevier Masson

http:// www.elsevier-masson.fr/

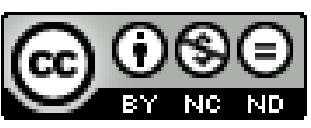


A comparison of animal personality and coping styles in the red junglefowl

Running headline: Comparing animal personality and coping styles

Keywords: boldness, exploration, Gallus gallus, individual differences, stress coping 
There is an increased focus in biology on consistent behavioural variation. Several terms are used to describe this variation, including animal personality and coping style. Both terms describe among-individual consistency in behavioural variation, however they differ in the behavioural assays typically used, the expected distribution of response variables, and whether they incorporate variation in behavioural flexibility. Despite these differences, the terms are often used interchangeably. We conducted experiments using juvenile and adult red junglefowl (Gallus gallus) as subjects to explore the degree to which animal personality and coping styles overlap. We demonstrate that animal personality and coping styles can be described in this species, and that shyer individuals had more flexible responses, as expected for coping styles. Behavioural responses from both personality and coping style assays had continuous distributions, and were not clearly separated into two types. Behavioural traits were not correlated and, hence, there was no evidence of a behavioural syndrome. Further, behavioural responses obtained in personality assays did not correlate with those from coping style tests. Animal personality and coping styles are therefore not synonymous in the red junglefowl. We suggest that the terms animal personality and coping style are not equivalent and should not be used interchangeably. 
Several terms are used to describe consistent among-individual variation in animal behaviour, such as ‘animal personality’ (Dall, Houston \& McNamara, 2004; Sih, Bell, Johnson \& Ziemba, 2004a), 'coping style' (Koolhaas, et al., 1999), and 'temperament' (Boissy, 1995; Clarke \& Boinski, 1995; Réale, Reader, Sol, McDougall \& Dingemanse, 2007). Common features of animal personality and coping styles are that behavioural responses are expected to show consistent variation among and within individuals, and that this variation can be biologically meaningful. However, despite these similarities, there are fundamental differences in how behavioural variation and consistency are investigated, including the behaviours recorded, their distribution and the assays used to measure variation in behaviour.

\section{Defining animal personality and coping styles}

Animal personality is commonly defined as among-individual differences in behaviour that are consistent over time and/or across context (Dall, et al., 2004; Sih, et al., 2004a, Table 1). Wild and captive animals of both sexes have been studied in stressful and not stressful situations, and variation in activity, aggression, boldness, exploration, and sociability are described for multiple taxa (Gosling, 2001; Réale, et al., 2007; Carere \& Maestripieri, 2013). A coping style is typically defined as a set of correlated behavioural and physiological stress responses, which are consistent over time (Koolhaas, et al., 1999, Table 1). In contrast to studies of personality, laboratory male rodents have been the main focus of coping style studies (Carere, Caramschi \& Fawcett, 2010; but see e.g., Castanheira et al., 2015, Table 1). Coping styles differ somewhat from the broader definition of animal personality by describing behavioural strategies to handle stressful situations in which several behaviours are intercorrelated and correlate with physiological mechanisms, such as stress response and androgen levels (e.g., Koolhaas, et al., 1999, 2010; Cervantes \& Delville, 2007; Øverli, Sørensen, Pulman, Pottinger \& Korzan, 2007; 
Coppens, et al., 2010; Castanheira, et al., 2015). In contrast, physiological measures are rarely included in descriptions of personality, though a few studies relate variation in personality to stress physiology (Cockrem, 2007; Kralj-Fišer, Weiß \& Kotrschal, 2010; Baugh, Schaper, Hau, Cockrem \& Goede, 2012). Furthermore, personality traits can be inter-correlated and form 'behavioural syndromes’ (Sih, et al., 2004a, e.g., great tits, Parus major, Verbeek, Boon \& Drent, 1996), but do not necessarily do so (e.g., three-spined stickleback, Gasterosteus aculeatus, Dingemanse, et al., 2007; checkered pufferfish, Sphoerodies testdineus, Pleizier, Wilson, Shultz \& Cooke, 2015; fowl, Gallus gallus ssp., Favati, Leimar \& Løvlie, 2014a; Favati, Leimar, Radesäter \& Løvlie, 2014b; Favati, Zidar, Thorpe, Jensen \& Løvlie, 2016).

\section{Distribution of behavioural traits}

Contrary to the continuous distribution of most personality traits, coping styles were initially typically described as two distinct phenotypes; proactive and reactive, often with opposing behavioural and physiological features (for review, see Koolhaas, et al., 1999, 2010; Coppens, et al., 2010). It is often shown that reactive individuals respond passively by withdrawing from stressful situations ('conservation/withdrawal'), i.e., they try to avoid the stressor. Proactive individuals instead show an active approach ('fight/flight'), i.e., trying to counteract the stressor (for review, see Koolhaas, et al., 1999; Øverli, et al., 2007). Reactive individuals also tend to be more flexible and aware of environmental changes, whereas proactive individuals tend to be more rigid in their behaviour and easily form routines (e.g., mice, Mus musculus, Benus, et al., 1990; pigs, Sus scrofa, Bolhuis, Schouten, de Leeuw, Schrama \& Wiegant, 2004; great tits, Carere, Drent, Privitera, Koolhaas \& Groothuis, 2005, greylag geese, Anser anser, Kralj-Fišer, Scheiber, Blejec, Moestl \& Kotrschal, 2007).

\section{Behavioural flexibility}


Both theoretical models (Wolf, van Doorn \& Weissing, 2008; Coppens, et al., 2010) and empirical studies (e.g., mice, Benus, et al., 1990; pigs, Bolhuis, et al., 2004; rainbow trout, Oncorhynchus mykiss, Ruiz-Gomez, Huntingford, Øverli, Thörnqvist \& Höglund, 2011), support that behavioural flexibility is an important underlying aspect describing coping styles. Individuals that are less flexible are expected to be bolder and more proactive (Koolhaas, et al., 1999; Wolf, et al., 2008; Dingemanse, Kazem, Réale \& Wright, 2010). This has been observed, for example, in pigs where high resistance in the back test (describing proactive individuals) was negatively associated with behavioural flexibility (Bolhuis, et al., 2004), and in rainbow trout where proactive individuals were less flexible in reversal learning (Ruiz-Gomez, et al., 2011).

Behavioural flexibility has not traditionally been considered an important aspect of animal personality. More recently, individual plasticity has been discussed in relation to animal personality in the context of reaction norms (Dingemanse, et al., 2010, 2012) and responsiveness (Wolf, et al., 2008). Reaction norms describe individuals’ differential responses to changes in the environment (e.g., perceived risk of predation, Dingemanse, et al., 2010, 2012) and responsiveness describes flexible responses to varying situations (Wolf, et al., 2008). Both reaction norms and responsiveness differ from coping style research where flexibility in specific (often operant) tasks is assessed (see below). Behavioural flexibility is therefore possibly an important aspect of animal personality, although this possibility has not yet been well explored empirically.

\section{Behavioural assays}

Common personality assays include open field (arena without interior), novel arena (arena with interior encouraging exploration) and novel object tests (e.g., Walsh \& Cummins, 1976; 
Dingemanse, et al., 2007; Réale, et al., 2007; Carere \& Maestripieri, 2013; Carter, Feeney, Marshall, Cowlishaw \& Heinsohn, 2013). In these tests, the reaction of animals to novelty is measured.

Coping style assays can include the above, and also include relatively more stressful tests like responses to introduction of electric probes into the home pen (Sluyter, Korte, Bohus \& van Oortmerssen, 1996; Koolhaas, et al., 1999, 2010) and forced swim tests (Koolhaas, et al., 2010). Additionally,, a commonly used test is the back-test in which the individual is held on its back and resistance (Bolhuis, et al., 2004) or latency to remain in tonic immobility, if that is induced, is measured (Forkman, Boissy, Meunier-Salaün, Canali \& Jomes, 2007). However, the typical tests designed to capture coping strategies often include measures of responses to alteration of learnt tasks, often through maze or operant conditioning tests (Coppens, et al., 2010).

In a maze test, the animal is typically trained to move through a maze to obtain a reward, and after reaching a stable routine the maze is changed and the behavioural reaction of the animal is measured. For example, Benus (1990) performed a maze test, in which male mice from different selection lines responded very differently to a seemingly small change (turning the maze 90 degrees) . Individuals from a long attack latency line started to explore the maze carefully, whereas individuals from a short attack latency line seemed unaffected by the change. Latency to ignore a previously rewarded stimulus in favour of a previously unrewarded stimulus, during the transition between associative and reversal learning, is also used to measure behavioural flexibility and variation in coping (e.g., Pavlov, 1908/1941; Bolhuis, et al., 2004; Coppens, et al., 2010; Klanker, Feenstra \& Denys, 2013). In these types of tasks, less flexible individuals form routines and therefore are slow to investigate the previously unrewarded stimulus, whereas more flexible individuals investigate the previously unrewarded stimulus 
quickly.

Are the terms synonymous?

Despite these differences in the terminology and tests used for animal personality and coping style, there is an assumption that animal personality and coping style are describing the same, or at least a very similar phenomenon. This assumption can confuse comparisons and hamper our understanding of the observed variation. For example, spontaneous behaviour vs. conditioned responses in operant tests, and bimodal vs. continuous responses may have different underlying mechanisms, and selection may act differently on these traits. To our knowledge the relationship between behavioural responses obtained in personality assays and coping style tests have rarely been compared in animal models.

In the current study we exposed juvenile and adult red junglefowl (Gallus gallus) to behavioural assays used to describe variation in animal personality and coping styles. We focus on often studied and biologically relevant personality traits of boldness and exploration (Reale et al., 2007), and coping style tasks that capture fearfulness and behavioural flexibility, important aspects of coping style. If behavioural responses are clustered into coping styles, bolder, less fearful individuals that explore more should be less flexible, describing a proactive type. Shyer individuals who explore less should, in this scenario, be more flexible, describing reactive individuals (Koolhaas, et al., 1999, 2010; Coppens, et al., 2010). On the other hand, if boldness, exploration and behavioural flexibility are not related and instead describe separate behavioural traits, this fits better with the view that personality traits and coping styles are not synonymous in this species. We thus expect responses from the different tests to correlate if personality and coping styles are describing the same aspects of behaviour variation. However, this may not be the case. Our previous results show that boldness and exploration do not form a behavioural 
syndrome in this species (Favati et al., 2014a, b). We use both juveniles and adults, which enables investigation of general patterns of behavioural variation over ontogeny. Our previous results show that correlation within and among behavioural traits can change over the animal's lifetime (Favati et al., 2016). We may therefore observe differences in responses between juvenile and adult animals. Overall, this leads us to predict that the coping styles in the red junglefowl are not similar to coping styles described in rodents, and that personality and coping style are not the same in this species.

\section{Method}

\section{Animals and housing}

From March to September 2013, we used subjects from a population of captive, pedigree-bred red junglefowl, which is the ancestor of domesticated fowl (Fumihito, et al., 1994). Subjects were reared at Linköping University, Sweden, and have been bred in captivity since 1998 . The focal population has not been bred or used for commercial purposes, thus their behaviour is similar to that observed in wild red junglefowl (Collias \& Collias, 1967, Sawai, et al., 2010). Further, juveniles are precocial which enables the use of juveniles hatched in incubators, eliminating the influence of maternal affects after the egg has been laid.

Two samples of subjects totalling 100 individuals $\left(N_{\text {females }}=55, N_{\text {males }}=45\right)$ from 18 families ( $n_{\text {individuals per family }}=2$ - 9) were used in this study. Directly after hatching, subjects were individually marked with wing-tags to facilitate recognition. The juveniles were housed in same-age, mixed-sex groups in 1-3 indoor enclosures $\left(0.5-3.0 \mathrm{~m}^{2}\right)$ during the first set of behavioural test (see below). Eight weeks post-hatching and after these behavioural tests, the 
juveniles were transported to Wood-Gush the facility where adult birds are kept where they were housed in same sex groups (indoor + outdoor area: $3+3 \mathrm{~m}^{2}$ ). Thirteen individuals took part in another study at this stage and were thus excluded from further testing.

\section{Ethical notes}

The experiment was conducted according to ethical legislation in Sweden and according to an approved ethical permit from Linköping Ethical committee (ethical permit number 122-10). Both wild and domestic fowl are easily habituated to humans, enabling reduced stress during catching, handling, and behavioural observations. The experimental housing was either identical or very similar to that of stock housing and included environmental enrichment in the form of perches and wood shavings enabling dust bathing; ad libitum access to commercial poultry food and water. The light was on a 12/12 hour dark/light cycle. The health and wellbeing of birds at this facility is checked at least once per day. After participating in a test, subjects were released back into their home pens. Animals at the facility that are injured or sick are cared for or euthanised by trained gamekeepers, or the veterinarian. In the present study, no subjects were injured and no harmful manipulations were carried out. While behavioural assays can induce some stress this is considered mild. Further, we took additional measures to reduce stress (e.g., allowing social companions in their home pen and when familiarising themselves with the test arena, and providing rewards). Any bird that showed signs of distress was taken back to its home pen.

\section{Experimental setup}


All recorded behaviours were obtained on singly tested subjects, and all latencies recorded are in seconds. Table 2 presents details of and motivation for personality and coping style tests.

\section{Assays used for scoring personality in juveniles}

A total of 100 subjects were tested in novel arena, novel object and tonic immobility tests (see below) as juveniles at 4 and 6 weeks of age.

\section{Exposure to a novel arena}

To observe variation in exploration and activity in a situation where fear of novelty can conflict with tendency to explore (Steimer, la Fleur \& Schulz, 1997; Forkman, et al., 2007; Réale et al., 2007), subjects were exposed to a novel arena test (NA) (Table 2). Two identical arenas built out of plywood with a mesh roof and rubber floor measuring 114 x $76 \times 40 \mathrm{~cm}$ were used (see SI, Fig S1). Empty, familiar food and water containers were placed in the arenas to obscure the subjects' view of the arena and encourage exploration. Substrate and placement of containers were altered to keep the environment novel between the test occasions. For all subjects, wood shavings were used as substrate during the first test and shredded corrugated fibreboard during the second test (see SI, Fig S1a, b). The arena was divided into 6 imagined sections to observe the subjects' use of the arena. Behaviours scored were latency to move, latency to explore the entire arena, activity, vigilance and number of escape attempts (Favati et al., 2016; Zidar et al., 2017). Behaviours were scored by instantaneous sampling every 10 sec, observed directly via video-link to a screen, for the 10 min the test lasted.

\section{Exposure to a novel object}

Directly following the novel arena test, a novel object test was implemented. Novel objects are used to measure variation in object-related neophobia (Greggor, Thornton \& Clayton, 2015), or 
when the object resembles a predator, to measure variation in boldness (Réale, et al., 2007, Table 2). We used a plush toy measuring $15 \mathrm{~cm}$ in diameter and with $\sim 2 \mathrm{~cm}$ large yellow and black eyes, thus resembling a predator. Lights were turned off and the novel object was placed in the arena as far away from the subject as possible. To keep the object novel, it was changed between the two test occasions (yellow versus black/white coloured plush toy at 4 and 6 weeks respectively). The behaviours recorded were the same as described for the novel arena test above.

\section{Assays used for measuring coping style in juveniles}

\section{Responses to induced tonic immobility}

The tonic immobility test is used to measure fear responses in birds (Forkman, et al., 2007; similar to the back test in pigs, Bolhuis, et al., 2004, Table 2). To induce tonic immobility, subjects were placed on their backs in a V-shaped wooden cradle $(20 \times 10 \mathrm{~cm})$. A light pressure was applied to the subject's breast while also loosely holding a hand over their head for fifteen seconds. Thereafter the pressure was released and latency until the subject started to move its head was used to measure time spent in a tonic state ('latency to move after induced tonic immobility'). To reduce the influence of the observer on the duration of tonic immobility, the observer avoided direct eye contact with the subject. We used the same observer for all tonic immobility tests. If a subject was not induced into tonic immobility, i.e., started to move within 3 sec following the removal of the pressure by the hand, the procedure was repeated up to 3 times. If the subject was still not induced into tonic immobility after 3 attempts, the subject received a latency of 0 sec. If the subject stayed in tonic immobility for more than 10 min, they received a maximum latency of $600 \mathrm{sec}$ and the test was terminated. 
Measures of behavioural flexibility

To measure behavioural flexibility, we measured both individual behavioural responses to alteration of rewarded stimulus and responses to intramaze change (see below, Table 2).

\section{Response to alteration of rewarded stimulus}

From day 1 post-hatching all juveniles were first handled and familiarised with the test arena $(28 \times 18 \times 37 \mathrm{~cm}$, see Fig S2). Initially, to reduce stress due to social isolation, the juveniles were exposed to the arena in groups. Group size was then continuously decreased until each juvenile showed no sign of stress from being in the test arena on its own. To test associative learning speed, subjects $\left(N=62, N_{\text {females }}=35, N_{\text {males }}=27\right)$ took part in a series of trials three days post-hatching. Subjects were presented with two bowls; one blue and one green separated by a cardboard divide, and allowed to choose one bowl to investigate per trial. Subjects were randomly assigned to a group in which blue or green were associated with a food reward ( one third of a mealworm). The association was considered stable when 5 correct choices in a row were made. To explore variation in behavioural flexibility, after reaching this criterion the previously unrewarded stimulus was rewarded while the previously rewarded stimulus was left unrewarded. We recorded the latency for a juvenile to investigate the previously unrewarded stimulus after this alternation. One male did not learn the association and was excluded from the analysis.

\section{Response to intra-maze change}

As juveniles at five weeks old, subjects were initially taught the location of a food reward (a mealworm in a bowl) located $0.5 \mathrm{~m}$ after a turn in a U-shaped arena $(76 \times 114 \mathrm{~cm}$, SI, Fig S4a). A trail of mealworms was initially used to encourage the subject to move towards the final reward. During later training, the reward was only given in the final bowl. Training continued 
until the subject had clearly associated a specific route with the reward (i.e., moved directly to the reward after release 5 times in a row, without stopping or turning around). Training sessions lasted up to 15 minutes and subjects needed about 5 training sessions to learn the task. After the association had been established, the trained route was cut off and a shortcut was opened in the beginning of the arena (20 cm from the starting position, SI, Fig S4b). Latency to find a shortcut was used as a measure of behavioural flexibility. Six juveniles $\left(N_{\text {females }}=5, N_{\text {males }}=1\right)$ were not able to learn the location of the food reward (e.g., due to lack of motivation to eat mealworms), and data from these individuals were not included in further analyses.

For the associative- and reversal learning and maze tests, each session continued for as long as the subject was motivated to work for the reward ( $\sim 15 \mathrm{~min})$. If a training session ended before an association was formed, a new training session commenced after approximately one hour of rest.

\section{Assays used to describe personality in adult fowl}

When sexually mature, at around 5 months of age (Johnsen \& Zuk, 1998), all available adult individuals ( $N=87, N_{\text {females }}=45, N_{\text {males }}=42$ ) were tested again in the personality assays previously described for the juveniles.

\section{Exposure to a novel arena}

The novel arena $\left(2 \mathrm{~m}^{2}\right)$ had peat as substrate and empty, familiar food and water containers to encourage exploration (see SI, figure S1c). Subjects were calmly caught from their home pen with the light switched off. Behaviours were recorded through direct instantaneous sampling every $10 \mathrm{sec}$. 


\section{Exposure to a novel object}

As described for juveniles, the novel object was placed in the arena directly following the novel arena test. Again, latency to move, latency to explore the entire arena, activity, vigilance, and number of escape attempts, were recorded through direct instantaneous sampling every 10 sec.

\section{Assays used for scoring coping styles in adults}

\section{Responses to induced tonic immobility}

'Latency to move after induced tonic immobility was measured on adult fowl in the same manner as described above for the juveniles.

\section{Response to change of rewarded stimulus}

Sexually mature males fowl are not very motivated by food, while females readily work for food rewards. Retesting of behavioural flexibility in the operant task only included females $\left(N_{\text {females }}=45\right)$.

Females were trained to associate one of two patterns (either white background with black circle, or black background with white circle) with a reward (mealworm), hidden in a bowl underneath a lid marked with the relevant pattern. The patterns of black and white had the same proportions, and females were randomly assigned to be rewarded for either of the two stimuli. The associative learning ability of the subjects was tested through their participation in a series of trials where subjects were allowed to choose between two bowls with the relevant patterns. A die was used to determine whether the rewarded stimuli was positioned on the left or the right. The criterion for passing the associative learning stage was 5 correct choices in a row. All individuals got exactly 100 trials over 6 training sessions over 2 days to learn the association. 
Thirty-four females learnt the association and were exposed to the reversal learning test in which latency to explore a new colour cue was scored.

\section{Statistical analyses}

All analyses were conducted using R version 3.0.2 ( $\mathrm{R}$ developmental core team 2014).

To assess whether our response variables were bimodal or continuous, we visually inspected histograms.

For responses measured both at 4 and 6 weeks, mean value of the two were used for further analyses.

The variables obtained from the novel arena and novel object tests were reduced by the use of principal component analysis (PCA). We obtained differentially weighted scores using the regression approach with the R-package psych and the function principal (Revelle, 2016). Numbers of principal components were chosen by a combination of visual inspection of screeplots, parallel analysis and examination of the components interpretability. For both the juvenile $(r=-0.28)$ and adult $(r=0.04)$ data, components were weakly correlated (allowing for orthogonal rotation, varimax, of the principal components). Two components were obtained for juveniles. PC1 was interpreted as describing individuals that are more vs. less active and that explore more vs. less, and PC2 as describing individuals that are more vs. less shy and fearful (Table 3). For adult fowl, two components were obtained. PC1 was interpreted as describing females that were more vs. less shy and fearful, PC2 as describing individuals that are more vs. less active and that explore more vs. less (Table 3). 
Repeatability of personality traits was evaluated using the 'rtpR' package (Nakagawa \& Schielzeth, 2010; Nakagawa, Schielzeth \& Stoffel, 2016). Histograms were assessed and depending on distribution, models were fitted for either Gaussian, Bimodal or Poisson distributed traits. This resulted in a linear mixed model ( $\mathrm{lmm}$ ) method for activity and vigilance for juveniles tested at 4 and 6 weeks of age, and for vigilance when comparing juveniles to adults. To compare activity between juveniles and adults we used a generalised linear mixed model (glmm) and log link function. Furthermore, for latencies and count data we use a glmm and log link function. Because our measure of latency to explore the entire arena included many max-values (i.e., latencies of $600 \mathrm{sec}$ ) we analysed this as a binary trait (i.e., explored only parts of the arena vs. explored the entire arena) with a glmm and log link function.

To test whether our response variables that were obtained in coping style tests were interrelated, Pearson's correlation tests on our transformed variables (see below) were carried out.

To explore the relationship between behavioural responses from personality assays and measures from coping style assays, we fitted general linear mixed effects models using the Rpackage 'Ime4' (Bates, Maechler, Bolker \& Walker, 2015). For the juvenile data, models included the fixed factors: sex (Male, Female), PC1, PC2, and the random effect family to account for sibling relatedness. For the adult data, models included the fixed effects PC1 and PC2 as well as family as a random effect. To meet the assumption of normality we selected suitable transformations for our response variables using Box-Cox. Normality was evaluated statistically with Shapiro-Wilk normality test. In cases were normality was not met we used $\sqrt{ }$ $(\chi)$ transformation for latency to move after induced tonic immobility for juveniles, and $\log (\chi)$ for latency to explore a new colour cue for both juveniles and adults, as well as for latency to 
find a shortcut in the spatial task after reconfiguration of the maze, and for latency to move after induced tonic immobility for adults.

\section{Results}

\section{Relationships between behavioural responses}

Repeatability of responses obtained in personality assays

Repeatable behavioural responses are a pre-requisite for determining whether a personality trait is being measured. We observed high repeatability for all behaviours scored in personality assays between 4 and 6 weeks of age (Table 4). Repeatability for behaviours scored in personality assays was low when comparing behaviour in juveniles at 4 weeks to adults (Table 4).

Relationship among responses obtained in coping style assays

Coping styles are expected to include intercorrelated traits. For juveniles, our two measures of flexibility, latency to explore a new colour cue and latency to find a shortcut in the spatial task, showed a non-significant positive correlation (Pearson's $r=0.22, N=55$, $\mathrm{df}=53, P=0.11$, Fig 1a). Because of this relationship, only the response to the change of rewarded stimuli was measured in adults. There was a negative correlation between latency to move after induced tonic immobility and latency to explore a new colour cue (Pearson's $r=-0.32, N=61$, $\mathrm{df}=59$, $P=0.01$, Fig $1 \mathrm{~b})$. However, there was no clear relationship between latency to move after induced tonic immobility and latency to find a shortcut in the spatial learning task (Pearson's $r$ $=-0.08, N=56, \mathrm{df}=54, P=0.58$, Fig $1 \mathrm{c})$. 
Similar to juveniles, latency to move after induced tonic immobility correlated negatively with latency to explore a new colour cue for adult females (Pearson's $r=-0.35, N=34$, $\mathrm{df}=32, P$ $=0.04$, Fig 1d).

\section{Distribution of behavioural responses}

Behavioural responses from both personality assays and coping style assays were continuous, for both juveniles (Fig 2) and adults (Fig 3). Thus, we did not observe a clear bimodal distribution as described for coping styles in rodents (Koolhaas, et al., 2010).

\section{Comparing animal personality and coping styles for juveniles}

Responses to induced tonic immobility

In juveniles, 'latency to move after induced tonic immobility' was not associated with sex $\left(\chi^{2}{ }_{1}\right.$ $=0.26, P=0.61$, Fig $4 \mathrm{a}, \mathrm{b})$, or any of the personality measures taken $\left(\mathrm{PC} 1: \chi^{2}{ }_{1}=0.01, P=0.91\right.$, Fig 4a; PC2: $\chi^{2}{ }_{1}=0.04, P=0.83$, Fig 4b).

Response to alteration of rewarded stimulus

'Latency to explore a new colour cue' did not differ between the sexes $\left(\chi^{2}{ }_{1}=2.74, P=0.10\right.$, Fig 4c, d), and was not associated with personality in terms of exploration (PC1: $\chi^{2}{ }_{1}=0.02, P=$ 0.88, Fig 4c), but was positively related to personality in terms of how shy and fearful a juvenile was (PC2: $\chi^{2}{ }_{1}=6.80, P=0.009$, Fig $\left.4 \mathrm{~d}\right)$. 
'Latency to find a shortcut' was not explained by $\operatorname{sex}\left(\chi^{2}{ }_{1}=0.72, P=0.40\right.$, Fig $\left.4 \mathrm{e}, \mathrm{f}\right)$, or any of the personality measures taken (PC1: $\chi^{2}{ }_{1}=0.80, P=0.37$ Fig 4e; PC2: $\chi^{2}{ }_{1}=0.21, P=0.65$, Fig 4f).

\section{Comparing animal personality and coping styles in adults}

Responses to induced tonic immobility

For adults, 'latency to move after induced tonic immobility' was not associated with sex $\left(\chi^{2}{ }_{1}=\right.$ 1.34, $P=0.25$, Fig 5a, b), or any of the personality measures taken $\left(\mathrm{PC} 1: \chi^{2}{ }_{1}=0.60, P=0.44\right.$, Fig 5a; PC2: $\chi^{2}{ }_{1}=0.28, P=0.60$, Fig $\left.5 b\right)$.

Behavioural responses to change of rewarded stimulus

In adult females, 'latency to explore a new colour cue' was not explained by any of the personality measures taken (PC1: $\chi^{2}{ }_{1}=0.51, P=0.48$, Fig 5c; PC2: $\chi^{2}{ }_{1}=0.21, P=0.65$, Fig $5 d)$.

\section{Discussion}

We show that behavioural responses in red junglefowl can describe variation in personality and coping, respectively. The behavioural responses measured in personality assays were not strongly related to behavioural responses measured in coping style assays. We observed the same pattern in both juveniles and adults. This suggests that personality and coping style capture different aspects of behavioural variation in this species. We therefore suggest that the two terms describing consistency in behavioural responses are not necessarily equivalent and should not be used as synonyms. 
Animal personality has been described in a range of species (Gosling, 2001; Carere \& Maestripieri, 2013). Consistency of among-individual variation in behavioural traits is a requisite for terming behavioural traits personality. This is commonly achieved by investigating the same or functionally similar behavioural responses, over time and/or across context. We observed among-individual consistency in the measured behaviours obtained from established personality assays (e.g., Dingemanse, et al., 2007; Réale, et al., 2007; Carere \& Maestripieri, 2013; Carter, et al., 2013). Specifically, we show that in red junglefowl, the gradients explaining variation in exploration and shyness-boldness are consistent in juveniles, although not from juvenile to adulthood. Our results are consistent with our previous studies in the species (Favati, et al., 2014a, b), including members of the same population (Favati, et al., 2016; Zidar, et al., 2017), and capture two gradients investigated and observed in other species (Réale, et al., 2007). The exploration and shyness gradients did not correlate, i.e., they did not form a behavioural syndrome. This is also in accordance with results from our previous studies in this species (Favati, et al., 2014a, 2016).

To describe variation in coping style, correlated stress responses are investigated (Koolhaas, et al., 1999, 2010; Coppens, et al., 2010). Thus, we expect that the interrelatedness between behavioural responses is similar over time (Koolhaas, et al., 1999). We explored variation in coping styles in our population of red junglefowl by exposing juveniles and adults to several tests similar to the ones typically used to score coping style in other species (Benus, 1990; Hessing, et al., 1993, Hessing, Hagelsø, Schouten, Wiepkema \& van Beek, 1994; Erhard \& Mendl, 1999; Erhard, Mendl \& Christiansen, 1999; Forkman, et al., 2007; Carere, et al., 2010; Castanheira, et al., 2015). Specifically, we investigated behavioural flexibility and fearfulness, traits that have been previously associated with stress response and thus coping style (Koolhaas et al. 2010). We found that these behavioural responses were, to some extent, interrelated across 
these tests. We show that juveniles that were fast to alter their response to a previously unrewarded stimulus after a previously rewarded stimulus became unrewarded were more fearful in a tonic immobility test as both juveniles and adults. Further, in juveniles, the latency to explore a previously unrewarded colour cue correlated positively, although not significantly, with latency to find the shortcut in an altered maze, showing a similar response to alteration of established associations. The relationship between behavioural flexibility and fearfulness is also what one would predict if these traits describe coping styles; reactive and fearful individuals showed higher behavioural flexibility, while proactive and bold individuals showed a more rigid, routine-like behaviour (Koolhaas, et al., 1999, 2010; Coppens, et al., 2010). This suggests that the responses recorded in these tests described variation in coping styles in this species. Our results are thus similar to what has been found in rodents, and in non-rodent species, such as pigs (Bolhuis, et al., 2004) and fish (Castanheira, et al., 2015). For example, in pigs, resistance in a back-test was negatively associated with behavioural flexibility (Bolhuis, et al., 2004). Additionally, lines of high and low responsive rainbow trout showed differences in behavioural flexibility and routine formation, which is what one would expect for different coping styles (Ruiz-Gomez, et al., 2011).

On the other hand, in the literature originally describing variation in coping styles, from studies conducted primarily on rodents, a dichotomy is described. Proactive and reactive individuals showed responses that were distinct from each other (Koolhaas, et al., 1999, 2010) and the distributions of the recorded behavioural responses were bimodal, corresponding to these distinct types (Koolhaas, et al., 2010, but see discussion in Castanheira, et al., 2015). However, in the red junglefowl, we found no such bimodality in coping style. It is still uncertain whether species other than rodents show these distinct response patterns, and it seems like responses obtained can be called coping styles even when not clearly bimodal (e.g., Koolhaas, et al., 
2010). Indeed, similar to us, several studies have failed to observe bimodal coping styles in their study subjects (e.g., Hessing, et al., 1993; Hessing, et al., 1994; Forkman, Furuhaug \& Jensen, 1995; Janczak, Pedersen \& Bakken, 2003; Jensen, Forkam, Thodberg \& Köster, 1995; Réale, et al., 2010; Castanheira, et al., 2015). Additionally, in contrast to reactive rodents being more behaviourally flexible, in some species proactive individuals have been found to be more behaviourally flexible (Frost, Winrow-Giffen, Ashley \& Sneddon, 2007; Basic, Winberg, Schjolden \& Höglund, 2012). Therefore, the generality of the distinction between proactive and reactive types is not totally clear and seems to vary depending on species. Finally, it is possible, through bidirectional selection, to convert a population that shows continuous responses to one that shows bimodal responses. This has been demonstrated in the great tit, an important model for consistent among and within individual differences in behavioural responses (Verbeek, et al., 1994; Nicolaus, et al., 2015).

In our study, responses describing animal personality and those describing coping styles did not clearly correlate. The exception to this was that bold and less fearful juveniles were more likely to modify their behaviour when exploring a previously unrewarded colour cue. The personality gradient explaining most variation in our data, exploration, was not associated with any of the responses obtained in coping style tests, either in juveniles or in adult females. Further, boldness and fearfulness did not relate to behavioural flexibility. Our results suggest that animal personality and coping styles are not overlapping in our species. This is similar to what has been found in checkered pufferfish where behavioural responses in personality assays did not correlate with measures of a physiological stress response, i.e., cortisol level (Pleizier, et al., 2015). One other possibility is that the way individuals cope with stressors, as described for coping styles, is a personality axis in itself. In great tits, traits typically used to describe variation in personality, including exploration speed and risk-taking, are included in the term coping style 
(e.g., Nicolaus, et al., 2015). Further, responses measured in tests used to describe animal personality and coping styles usually correlate in great tits (e.g., Verbeek, et al., 1994). This differs from what we observe in red junglefowl. A difference between great tits and red junglefowl is thus that personality gradients form behavioural syndromes in great tits, but do not seem to do so in red junglefowl (Favati, et al., 2014a, 2016). There are, however, a limited number of studies that compare variation in animal personality and coping styles, and so we do not know the degree to which these findings would generalise across species.

Behavioural flexibility is an important part of coping styles (Koolhaas, et al., 1999), but has not commonly been included in personality. We demonstrate that behavioural flexibility can predict variation in animal personality since variation in fearfulness and shyness-boldness was predicted by differences in behavioural flexibility in reaction to a change in an environmental cue and alteration of rewarded cue. Our results thus seem to capture a fundamental link between behavioural flexibility and personality. Behavioural flexibility (or responsiveness) has been discussed as complementary to animal personality (Wolf, et al., 2008; Dingemanse, et al., 2010, 2012), or as a separate personality gradient (Carter, Goldizen \& Heinsohn, 2012). Personalitydependent differences in behavioural flexibility have previously been observed in other species (e.g., Herborn, Heidinger, Alexander \& Arnold, 2014; Mathot, Wright, Kempenaers \& Dingemanse, 2012), including bird species (Titulaer, van Kees \& Naguib, 2012; Brust, Wuerz \& Krüger, 2013; Guillette, Reddon, Hoeshele \& Sturdy, 2011; Guillette, Hahn, Hoeschele, Przyslupski \& Sturdy, 2014) but the connection between personality and behavioural flexibility requires further exploration. Neuroendocrinology could potentially cause constraints in behavioural flexibility and thus explain why certain personality traits are observed (Sih, Bell \& Johnson, 2004b, Dall, et al., 2004, reviewed in Castanheira, et al., 2015). Exploration of the relationship between neuroendocrinology and personality is therefore also needed. 
Overall, in the red junglefowl, personality and coping styles describe different aspects of behavioural variation, and caution is warranted when using these terms in general. Nevertheless, the extensive work on the physiology and mechanisms involved in coping styles has played an important role in inspiring and stimulating work on how these mechanisms (e.g., corticosteroids, Cockrem, 2007; Baugh, et al., 2012; polymorphisms in dopamine receptor D4, DRD4, Fidler, et al., 2007) Finally, work on the fitness and adaptive value of observed variation in animal personality can potentially improve our understanding of coping style.

\section{Conclusions}

Our results suggest that the terms animal personality and coping style should not be used as synonyms, but should rather be used as separate terms that describe different aspects of consistent behavioural responses in animals. Thus, although biologists have grasped the importance of phenotypic variation in behaviour within species and populations, the generality of patterns describing different domains of personality and coping styles across species still remains unclear. Further, our results confirm that variation in personality can be linked to individual variation in behavioural flexibility.

\section{References}

Basic, D., Winberg, S., Schjolden, J. \& Höglund, E. (2012). Context-dependent responses to novelty in Rainbow trout (Oncorhynchus mykiss), selected for high and low post-stress cortisol responsiveness. Physiology \& Behavior, 105, 1175-1181. DOI 10.1016/j.physbeh.2011.12.021

Bates, D., Maechler, M., Bolker, B. \& Walker, S. (2015). Fitting Linear Mixed-Effects Models Using lme4. Journal of Statistical Software, 67(1), 1-48. DOI 10.18637/jss.v067.i01

Baugh, A. T., Schaper, S. V., Hau, M., Cockrem, J. F. \& Goede, de P. (2012). Corticosterone 
responses differ between lines of great tits (Parus major) selected for divergent personalities. General and Comparative Endocrinology, 175, 488-494. DOI 10.1016/j.ygcen.2011.12.012

Benjamini, Y. \& Hochberg, Y. (1995). Controlling the False Discovery Rate: A Practical and Powerful Approach to Multiple Testing. Journal of the Royal Statistical Society. Series B, 57, 289-300. URL http://www.jstor.org/stable/2346101

Benus, R. F., Bohus, B., Koolhaas, J. M. \& van Oortmerssen, G. A. (1989). Behavioural strategies of aggressive and non-aggressive male mice in active shock avoidance. Behavioural Processes, 20, 1-12. DOI 10.1016/0376-6357(89)90008-9

Benus, R. F., Bohus, B., Koolhaas, J. M. \& van Oortmerssen G. A. (1991). Heritable variation in aggression as a reflection of individual coping strategies. Experientia, 47, 10081019. DOI 10.1007/BF01923336

Benus, R. F., den Daas, S., Koolhaas, J. M. \& van Oortmerssen, G. A. (1990). Routine Formation and Flexibility in Social and Non-Social Behaviour of Aggressive and NonAggressive Male Mice. Behaviour, 112, 176-193. URL http://www.jstor.org/stable/4534836

Benus, R. F., Koohlhaas, J. M. \& van Oortmerssen, G. A. (1987). Individual differences in behavioural reaction to a changing environment in mice and rats. Behaviour, 100, 105122. DOI 10.1163/156853987X00099

Boissy, A. (1995). Fear and fearfulness in animals. The Quarterly Review of Biology, 70, 165191. URL http://www.jstor.org/stable/3036241

Bolhuis, J. E., Schouten, W. G. P., de Leeuw, J. A., Schrama, J. W. \& Wiegant, V. M. (2004). Individual coping characteristics rearing conditions and behavioural flexibility in pigs. Philosophical Transactions of the Royal Society B, 152, 351-360. DOI 10.1016/j.bbr.2003.10.024 
Brust, V., Wuerz, Y. \& Krüger, O. (2013). Behavioural flexibility and personality in zebra finches. Ethology, 119, 559-569. DOI 10.1111/eth.12095

Carere, C., Caramaschi, D. \& Fawcett, T. W. (2010). Covariation between personalities and individual differences in coping with stress: Converging evidence and hypotheses. Current Zoology, 56, 728-740.

Carere, C., Drent, P. J., Privitera, L., Koolhaas, J. M. \& Groothuis, T. G. G. (2005). Personalities in great tits, Parus major: stability and consistency. Animal Behaviour, 70, 795e805.

Carere, C. \& Maestripieri, D. (2013). Animal personalities: Behaviour, Physiology, and Evolution. Chicago and London: The University of Chicago press.

Carter, A. J., Feeney, W. E., Marshall, H. H., Cowlishaw, G. \& Heinsohn, R. (2013). Animal personality: what are behavioural ecologists measuring? Biological Reviews, 88, 465475. DOI 10.1111/brv.12007

Carter, A., Goldizen, A. \& Heinsohn, R. (2012). Personality and placticity: temporal behavioural reaction norms in a lizard, the Namibian rock agama. Animal Behaviour, 84, 471-477. DOI 10.1016/j.anbehav.2012.06.001

Castanheira, M. F., Conceição, L. E. C., Millot, S., Rey, S., Bégout, M-L., Dmasgård, B., Kristiansen, T., Höglund, E., Øyvind, Ø. \& Martins, C. I. M. (2015). Coping styles in farmed fish: consequences for aquaculture. Reviews in Aquaculture, 7, 1-19. DOI 10.1111/raq.12100

Cervantes, M. C. \& Delville, Y. (2007). Individual differences in offensive aggression in golden hamsters: a model of reactive and impulsive aggression? Neuroscience, 150, 511-521. DOI 10.1016/j. neuroscience.2007.09.034

Clarke, A. S. \& Boinski, S. (1995). Temperament in nonhuman-primates. American Journal of Primatology, 37, 103-125. DOI 10.1002/ajp.1350370205 
Cockrem, J. F. (2007). Stress, corticosterone responses and avian personalities. Journal of Ornithology, 148, 169-178. DOI 10.1007/s10336-007-0175-8

Collias, N. E. \& Collias, E. C. (1967). A field study on the red jungle fowl in northcentral India. The Condor, 69, 360-386. DOI 10.2307/1366199

Coppens, C. M., de Boer, S. F. \& Koolhaas, J. M. (2010). Coping styles and behavioural flexibility: towards underlying mechanisms. Proceedings of the Royal Society B, 365, 4021-4028. DOI 10.1098/rstb.2010.0217

Dall, S. R. X., Houston, A. I. \& McNamara, J. M. (2004). The behavioural ecology of personality: Consistent individual differences from an adaptive perspective. Ecology Letters, 7, 734-739. DOI 10.1111/j.1461-0248.2004.00618.x

Dingemanse, N. J., Bouwman, K. M., van de Pol, M., van Overveld, T., Patrick, S. C., Matthysen, E. \& Quinn, J. L. (2012). Variation in personality and behaviour plasticity across four populations of great tit Parus major. Journal of Animal Ecology, 81, 116126. DOI 10.1111/j.1365-2656.2011.01877.x

Dingemanse, N. J., Wright, J., Kazem, A. J. N., Thomas, D. K., Hickling, R. \& Dawnay, N. (2007). Behavioural syndromes differ predictably between 12 populations of threespined stickleback. Journal of Animal Ecology, 76, 1128-1138. DOI 10.1111/j.13652656.2007.01284.x

Dingemanse, N. J., Kazem, A. J. N., Réale, D. \& Wright, J. (2010). Behavioural reaction norms: animal personality meets individual plasticity. Trends in Ecology \& Evolution, 25, 81-89. DOI 10.1016/j.tree.2009.07.013

Erhard, H. W. \& Mendl, M. (1999). Tonic immobility and emergence time in pigs - more evidence for behavioural strategies. Applied Animal Behaviour Science 61, 227-37. DOI 10.1016/S0168-1591(98)00196-8

Erhard, H. W., Mendl, M. \& Christiansen, S. B. (1999). Individual differences in tonic 
immobility may reflect behavioural strategies. Applied Animal Behaviour Science, 64, 31-46. DOI 10.1016/S0168-1591(99)00028-3

Favati, A., Leimar, O. \& Løvlie, H. (2014a). Personality predicts social dominance in male domestic fowl. PLoS One, 9, e103535. DOI 10.1371/journal.pone.0103535

Favati, A., Leimar, O., Radesäter, T. \& Løvlie, H. (2014b). Social status and personality: stability in social state can promote consistency of behavioural responses. Proceedings of the Royal Society B, 281, 20132531. DOI 10.1098/rspb.2013.2531

Favati, A., Zidar, J., Thorpe, H., Jensen, P. \& Løvlie, H. (2016). The ontogeny of personality traits in the red junglefowl, Gallus gallus. Behavioral Ecology, 27, 484-493 DOI 10.1093/beheco/arv177

Fidler, A. E., van Oers, K., Drent, P. J., Kuhn, S., Mueller, J. C. \& Kempenaers, B. (2007). Drd4 gene polymorphisms are associated with personality variation in a passerine bird. Proceedings of the Royal Society B, 274, 1685-1691. DOI 10.1098/rspb.2007.0337

Forkman, B., Boissy, A., Meunier-Salaün, M-C., Canali, E. \& Jomes, R. B. (2007). A critical review of fear tests used on cattle, pigs, sheep, poultry and hourses. Physiology \& Behavior, 92, 340-374. DOI 10.1016/j.physbeh.2007.03.016

Forkman, B., Furuhaug, I. L. \& Jensen, P. (1995). Personality, coping patterns, and aggression in piglets. Applied Animal Behaviour Science, 45, 31-42. DOI 10.1016/01681591(95)00601-N

Frost, A. J., Winrow-Giffen, A., Ashley, P. J. \& Sneddon, L. U. (2007). Plasticity in animal personality traits: does prior experience alter the degree of boldness? Proceedings of the Royal Society B, 274, 333-339. DOI 10.1098/rspb.2006.3751

Fumihito, A., Miyake, T., Sumi, S., Takada, M., Ohno, S. \& Kondo, N. (1994). One subspecies of the red junglefowl (Gallus gallus gallus) suffices as the matriarchic ancestor of all domestic breeds. Proceedings of the National Academy of Sciences of the United States 
of America, 91, 12505-12509. URL http://www.jstor.org/stable/2366370

Gosling, S. D. (2001). From mice to men: What can we learn about personality from animal research? Psychological Bulletin, 127, 45-86. DOI 10.1037/0033-2909.127.1.45

Groothuis, T. G. \& Carere, C. (2005). Avian personalities: characterization and epigenesist. Neuroscience \& Biobehavioral Reviews, 29, 137-150. DOI 10.1016/j.neubiorev.2004.06.010

Greggor, A. L., Thornton, A. \& Clayton, N. S. (2015). Neophobia is not only avoidance: improving neophobia tests by combining cognition and ecology. Current Opinion in Behavioral Sciences, 6, 82-89. DOI 10.1016/j.cobeha.2015.10.007

Guillette, L. M., Reddon, A. R., Hoeshele, M. \& Sturdy, C. B. (2011). Sometimes slower is better: slow-exploring adults are more sensitive to changes in a vocal discrimination task. Proceedings of the Royal Society B, 278, 767-773. DOI 10.1098/rspb.2010.1669

Guillette, L. M., Hahn, A. H., Hoeschele, M., Przyslupski, A-M. \& Sturdy, C. B. (2014). Individual differences in learning speed, performance accuracy and exploration behaviour in black-chapped chickadees. Animal Cogntion, 18, 165. DOI 10.1007/s10071-014-0787-3

Henry, J. P. \& Stephens, P. M. (1977). Stress, Health and the Social Environment: Sociobiological Approach to Medicine. New York: Springer Verlag.

Herborn, K. A., Heidinger, B. J., Alexander, L., Arnold, K. E. (2014). Personality predicts behavioral flexibility in a fluctuating, natural environment. Behavioral Ecology, 25, 1374-1379. DOI 10.1093/beheco/aru131

Hessing, M. J. C, Hagelsø, A. M., van Beek, J. A. M., Wiepkema, P. R., Schouten, W. P. G. \& Krukow, R. (1993). Individual behavioural characteristics in pigs. Applied Animal Behaviour Science, 37, 285-95. DOI 10.1016/0168-1591(93)90118-9

Hessing, M. J. C., Hagelsø, A. M., Schouten, W. G. P., Wiepkema, P. R. \& van Beek, J. A. 
M. (1994). Individual behavioral and physiological strategies in pigs. Physiology \& Behavior, 55, 39-46. DOI 10.1016/0031-9384(94)90007-8

Janczak, A. M., Pedersen, L. J. \& Bakken M. (2003). Aggression, fearfulness and coping styles in female pigs. Applied Animal Behaviour Science, 81, 13-28. DOI 10.1016/S01681591(02)00252-6

Jensen, P., Forkam, B., Thodberg, K. \& Köster, E. (1995). Individual variation and consistency in piglet behaviour. Applied Animal Behaviour Science, 45, 43-52. DOI 10.1016/01681591(95)00591-F

Johansen, T. S. \& Zuk, M. (1998). Parasites, Morphology, and Blood Characters in Male Red Jungle Fowl during Development. The Condor, 100, 749-752. DOI 10.2307/1369760

Klanker, M., Feenstra, M. \& Denys, D. (2013). Dopaminergic control of cognitive flexibility in humans and animals. Frontiers in Neroscience, 7, 1-24. DOI 10.3389/fnins.2013.00201

Koolhaas, J. M., de Boer, S. F., Coppens, C. M. \& Buwalda, B. (2010). Neuroendocrinology of coping styles: Towards understanding the biology of individual variation. Frontiers in Neuroendocrinology, 31, 307-321. DOI 10.1016/j.yfrne.2010.04.001

Koolhaas, J. M., Korte, S. M., De Boer, S. F., van Der Vegt, B. J., van Reenen, H., Hopster, C. G., De Jong, I. C., Ruis, M. A. W. \& Blokhuis, H. J. (1999). Coping styles in animals: current status in behavior and stress-physiology. Neuroscience \& Biobehavioral Reviews, 23, 925-935. DOI 10.1016/S0149-7634(99)00026-3

Kralj-Fišer, S., Scheiber, I. B. R., Blejec, A., Moestl, E. \& Kotrschal, K. (2007). Individualities in a flock of free-roaming greylag geese: behavioral and physiological consistency over time and across situations. Hormones \& Behavior, 51, 239-248. DOI 10.1016/j.yhbeh.2006.10.006 
Kralj-Fišer, S., Weiß, B. M. \& Kotrschal, K. (2010). Behavioural and physiological correlates of personaliy in greylag geese (Anser anser), Journal of Ethology, 28, 363-370. DOI 10-1007/s10164-009-0197-1

Mathot, K. J., Wright, J., Kempenaers, B. \& Dingemanse, N. J. (2012). Adaptive strategies for managing uncertainty may explain personality-related differences in behavioural plasticity. Oikos, 121, 1009-1020. DOI 10.1111/j.1600-0706.2012.20339.x

Nakagawa, S., Schielzeth, H. \& Stoffel, M. (2016). rptR: Repeatability Estimation for Gaussian and Non-Gaussian Data. https://CRAN.R-project.org/package=rptR

Nakagawa, S. \& Schielzeth, H. (2010). Repetability for Gaussian and non-Gaussian data: a principal guide for biologists. Biological Reviews, 85, 935-956. DOI 10.1111/j.1469185X.2010.00141.X

Nicolaus, M., Mathot, K. J., Araya-Ajoy, Y. G., Mutzel, A., Wijmenga, J. J., Kempenaers, B. \& Dingemanse, N. J. (2015). Does coping style predict optimization? An experimental test in a wild passerine bird. Proceedings of the Royal Society B, 282, 20142405. DOI 10.1098/rspb.2014.2405

Oortmerssen van, G. A. \& Bakker, T. C. M. (1981). Artificial selection for short and long attack latencies in wild Mus musculus domesticus. Behavior Genetics, 11, 115-26. DOI 10.1007/BF01065622

Pavlov, I. P. (1908/1941). Lectures on conditioned reflexes. Volume two: Conditioned reflexes and psychiatry (W. H. Gantt, Trans.). London: Lawrence and Wishart Limited.

Pleizier, N., Wilson, A. D. M., Shultz, A. D. \& Cooke, S. J. (2015). Puffed and bothered: Personality, performance, and the effects of stress on checkered pufferfish. Physiology \& Behavior, 152, 68-78. DOI 10.1016/j.physbeh.2015.09.011 
R Development Core Team. (2014). R: A language and environment for statistical computing. R Foundation for Statistical Computing, Vienna, Austria Available at http://www.rproject.org/

Réale, D., Reader, S. M., Sol, D., McDougall, P. T. \& Dingemanse, N. J. (2007). Integrating animal temperament within ecology and evolution. Biological Reviews, 82, 291-318. DOI 10.1111/j.1469-185X.2007.00010.x

Revelle, W. (2016) psych: Procedures for Personality and Psychological Research, Northwestern University, Evanston, Illinois, USA. https://CRAN.Rproject.org/package=psych Version $=1.6 .9$

Ruiz-Gomez, M. L., Huntingford, F. A., Øverli, Ø., Thörnqvist, P-O. \& Höglund, E. (2011). Response to environmental change in rainbow trout selected for divergent stress coping styles. Phsysiology \& Behavior, 102, 217-322. DOI 10.1016/j.physbeh.2010.11.023

Sawai, H., Kim, H. L., Kuno, K., Suzuki, S., Gotoh, H., Takada, M., et al., (2010). The origin and genetic varation of domestic chickens with special reference to junglefowl Gallus g. gallus and G. varius. PLoS ONE, 5, e10639 DOI 10:1371/journal.pone.0010639

Sih, A., Bell, A. M., Johnson, J. C. \& Ziemba, R. E. (2004a). Behavioral syndromes: An integrative overview. The Quarterly Review of Biology, 79, 241-558. URL http://www.jstor.org/stable/10.1086/422893

Sih, A., Bell, A. \& Johnson, J. C. (2004b). Behavioural syndromes: an ecological and evolutionary owerview. Trends in Ecology \& Evolution, 19, 372-378. DOI 10.1016/j.tree.2004.04.009

Sluyter, F., Korte, S. M., Bohus, B. \& van Oortmerssen, G. A. (1996). Behavioral stress response of genetically selected aggressive and nonaggressive wild house mice in the shock-probe/defensive burying test. Pharmacology, Biochemistry \& Behavior, 54, 1136. DOI 10.1016/0091-3057(95)02164-7 
Steimer, T., la Fleur, S. \& Schulz, P. E. (1997). Neuroendocrine correlates of emotional reactivity and coping in male rats from the Roman high (RHA/Verh)- and low (RLA/Verh)-avoidance lines. Behavior Genetics, 27, 503-512. DOI 10.1023/A:1021448713665

Titulaer, M., van Kees, O. \& Naguib, M. (2012). Personality affects learning performance in difficult tasks in a sex-dependent way. Animal Behaviour, 83, 723-730. DOI 10.1016/j.anbehav.2011.12.020

Walsh, R. N. \& Cummins, R. A. (1976). The open-field Test - A critical review. Psychological Bulletin, 83, 482-504. DOI 10.1037/0033-2909.83.3.482

Verbeek, M. E. M., Drent, P. J., \& Wiepkema, P. R. (1994). Consistent individual differences in early exploratory behaviour of male great tits. Animal Behaviour, 48, 1113-1121. DOI 10.1006/anbe.1994.1344

Verbeek, M. E. M, Boon, A. \& Drent, P. J. (1996). Exploration, aggressive behaviour and dominance in pair-wise controntations of juvenile male grat tits. Behaviour, 133, 945963. URL: http://www.jstor.org/stable/4535403

Wolf, M., van Doorn, G. S. \& Weissing, F. J. (2008). Evolutionary emergence of responsive and unresponsive personalities. Proceedings of the National Academy of Sciences of the United States of America, 105, 15825-15830. URL: http://www.jstor.org/stable/25464500

Zidar, J., Sorato, E., Malmkvist, A-M., Jansson, E., Rosher, C., Jensen, J., Favati, A. \& Løvlie, H. (2017). Early experience affects adult personality in the red junglefowl: a role for cognitive stimulation? Behavioural Processes, 134, 78-86 DOI 10.1016/j.beproc.2016.06.003

Øverli, Ø., Sørensen., Pulman, K. G. T., Pottinger, T. G., Korzan, W., Summers, C. H. \& Nilsson, G. E. (2007). Evolutionary background for stress-coping styles: Relationships 
between physiological behavioural, and cognitive traits in non-mammalian vertebrates.

Neuroscience \& Biobehavioral Reviews, 31, 396-412. DOI 10.1016/j.neubiorev.2006.10.006 


\section{Tables and table legends}

2 Table 1. A comparison of similarities and differences between 'animal personality' and 'coping styles'.
Animal personality

\section{Coping style}

\section{Definition}

Among-individual behavioural differences

consistent over time and/or across context

$[1,2]$.

Subjects

Wild and captive species ranging from insects

to primates. Often research is conducted on

both sexes [1].

Tests/Assays used

\section{Exposure to unfamiliar or familiar}

environments, novel objects, simulated

predators, conspecifics $[1,2,9]$. Tonic

immobility test [6].

Response types
Primarily (but not only [3]), male rodents in a laboratory settings [4].

A coherent set of behavioural and physiological stress responses

[3]/characteristics [4] consistent over time [3] and over context [4].

Exposure to unfamiliar or familiar environments, novel objects, conspecifics, electrical probe [3]. Operant conditioning tasks, where after reaching a stable task performance, the reaction to a small change is studied $[5,4]$. Back-test or tonic immobility test $[7,8]$.

Spontaneous behaviour or reactions to changes in operant tests. Reactions to challenges [4] that allow different response patterns [5]. 
Response gradients Shyness-boldness, exploration-avoidance, activity, aggression, sociability [1].

\section{Response structure}

Quantitative, normally distributed responses along a unidimensional gradient ([1], but see ‘behavioural syndromes’ [9]).

Personality can partly be explained by

limitation in behavioural flexibility [2,9], and behavioural flexibility is complementary
Proactive-reactive [4], aggression [5], active-passive response to test situation, aspects of initiative [3]. quantitative aspects $[5,4]$, clustered in reactive - proactive phenotypes [3,5,4]. Responses are typically bimodal, or non-normal [3].

Behavioural flexibility is typically included as an important aspect [3,4, but see 10 for when it is not]. Reactive individuals are more flexible, and proactive individuals have stronger routine formation $[3,4]$.

$[11,12]$, or a separate personality gradient [13].

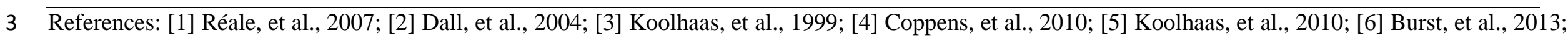

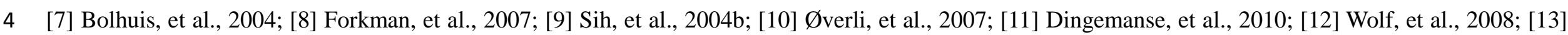

5 Carter, et al., 2012. 
6 Table 2. Overview of assays used, behaviour measured and whether they typically describe animal personality or coping styles.

\begin{tabular}{llc} 
Assay & Behaviour & Category \\
\hline Novel Arena & Activity, exploration [1-4] & Personality \\
Novel Object & Neophobia [1-4] & Personality \\
Tonic Immobility & Fearfulness [5] & Coping style \\
Change of rewarded stimulus & Behavioural flexibility [inspired by 6,7] & Coping style \\
Intra-maze change & Behavioural flexibility [8,9] & Coping style \\
\hline
\end{tabular}

13 References: [1] Favati, et al., 2014a; [2] Favati, et al., 2014b; [3] Favati, et al., 2016; [4] Zidar, et al., 2017; [5] Forkman, et al., 2007; [6] Titulaer, et al., 2012;

14 [7] Cervantes \& Delville, 2007; [8] Benus, et al., 1990; [9] Bolhuis, et al., 2004. 
Table 3. Principal component analysis of behavioural responses of red junglefowl juveniles in personality assays (Novel arena, NA, and Novel object, NO).

\begin{tabular}{|c|c|c|c|c|}
\hline & \multicolumn{2}{|c|}{ Juveniles } & \multicolumn{2}{|c|}{ Adults } \\
\hline Components & PC1 & PC2 & PC1 & PC2 \\
\hline Activity (NA) & 0.85 & 0.17 & 0.32 & -0.84 \\
\hline Vigilance (NA) & 0.71 & 0.42 & 0.79 & -0.22 \\
\hline Latency to move (NA) & -0.70 & 0.01 & 0.43 & 0.55 \\
\hline \multicolumn{5}{|l|}{ Latency to explore the entire arena } \\
\hline (NA) & -0.85 & -0.01 & -0.02 & $\mathbf{0 . 8 0}$ \\
\hline Vigilance (NO) & 0.16 & 0.85 & 0.68 & 0.06 \\
\hline Number of escape attempts (NO) & 0.01 & 0.79 & 0.71 & -0.03 \\
\hline After rotation sums of squares & 2.46 & 1.54 & 1.87 & 1.70 \\
\hline Proportion variance explained & 0.41 & 0.26 & 0.31 & 0.28 \\
\hline
\end{tabular}

31 For juveniles, PC1 is interpreted as describing individuals that are more vs. less active and that explore more vs. less, and PC2 as describing more vs. less shy

32 and fearful individuals. For adults, PC1 is interpreted as describing more vs. less shy and fearful individuals and PC2 as describing individuals that are more 

have values $> \pm 0.50 . N=100$ for juveniles, $N=87$ for adults. 
Table 4. Adjusted repeatability of personality traits of red junglefowl in a novel arena and novel object test, after controlling for sex.

\begin{tabular}{lcccc} 
& \multicolumn{2}{c}{$4-6$ weeks } & \multicolumn{2}{c}{ 4 weeks-adult } \\
\hline & $R$ & $95 \%$ CI & $R 5 \%$ CI \\
\hline Activity (NA) & 0.46 & $0.304-0.603$ & 0 & $0-0.21$ \\
Vigilance (NA) & 0.21 & $0.023-0.398$ & 0.02 & $0-0.23$ \\
Latency to move (NA) & 0.23 & $0.01-0.418$ & 0 & $0-0.21$ \\
Latency to explore the entire arena (NA) & 0.26 & $0-0.872$ & 0 & $0-0.115$ \\
Vigilance (NO) & 0.27 & $0.088-0.45$ & 0.12 & $0-0.238$ \\
Number of escape attempts (NO) & 0.59 & $0.391-0.727$ & 0 & $0-0.235$ \\
\end{tabular}

$46 \quad N=100$ for comparison between 4 and 6 weeks, $N=87$ for comparison between 4 weeks and when adult. 


\section{$47 \quad$ Figures and figure legends}

48 Figure 1. The relationship between behavioural responses scored in coping style assays for red 49 junglefowl. Black $=$ males, grey $=$ females.

50

51 Figure 2. Distribution of behavioural responses of red junglefowl juveniles obtained in 52 personality assays (a-f) and tests of coping style (g-i). NA = Novel Arena, NO = Novel Object, $53 \mathrm{TI}=$ Tonic Immobility, black $=$ males, grey $=$ females .

54

55 Figure 3. Distribution of behavioural responses of adult red junglefowl obtained in personality 56 assays (a-f) and tests of coping style (g-h). NA = Novel Arena, NO = Novel Object, $\mathrm{TI}=$ Tonic 57 Immobility, black $=$ males, grey $=$ females

58

59 Figure 4. Relationship between behavioural responses scored in personality assays and coping 60 style for red junglefowl juveniles. PC1 = more vs. less active and that explore more vs. less, 61 PC2 = more vs. less shy and fearful, black $=$ males, grey $=$ females.

62

63 Figure 5. Relationship between behavioural responses scored in personality assays and coping 64 style for adult red junglefowl. PC2 = more vs. less active and that explore more vs. less, black $65=$ males, grey $=$ females. 

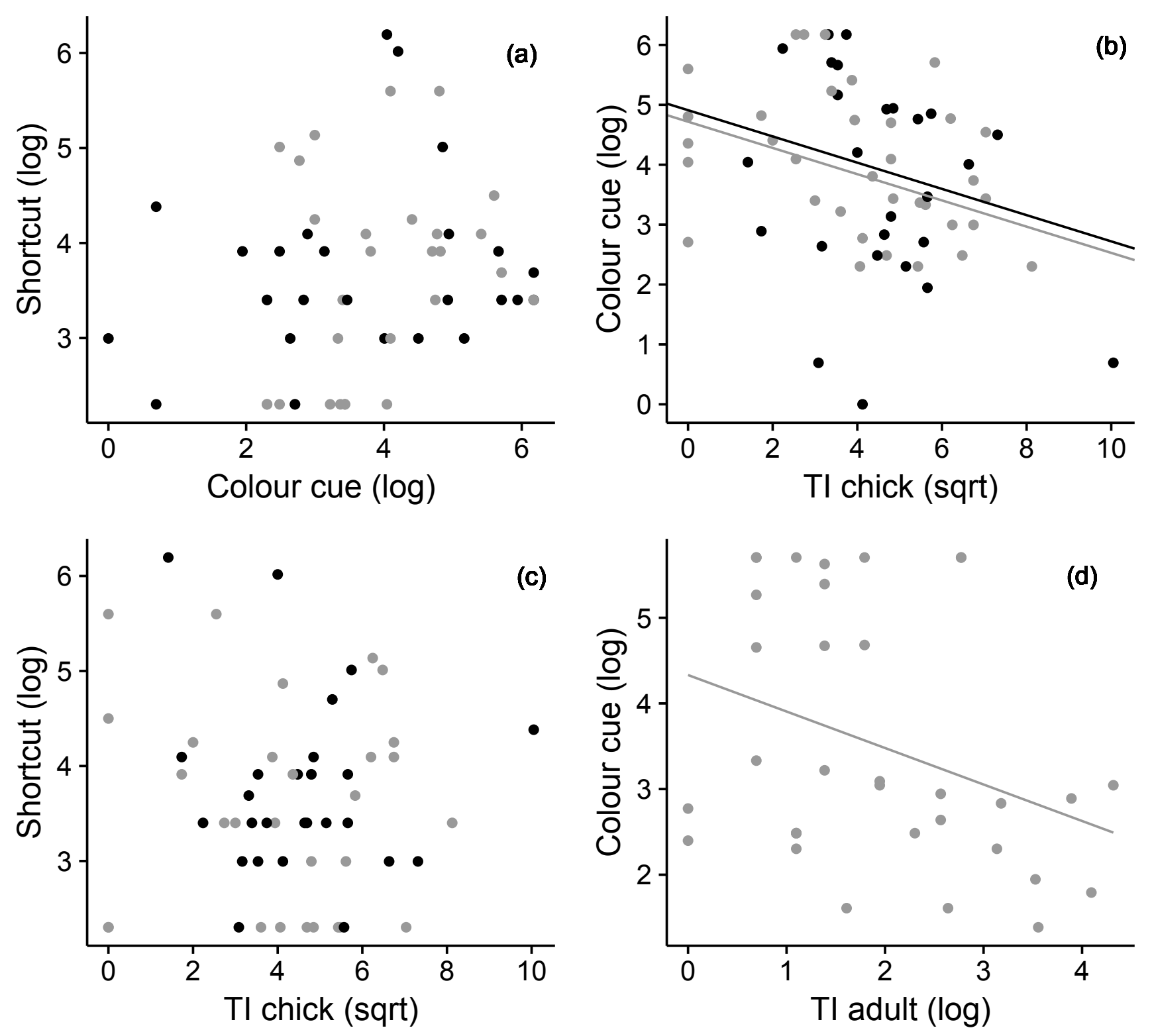


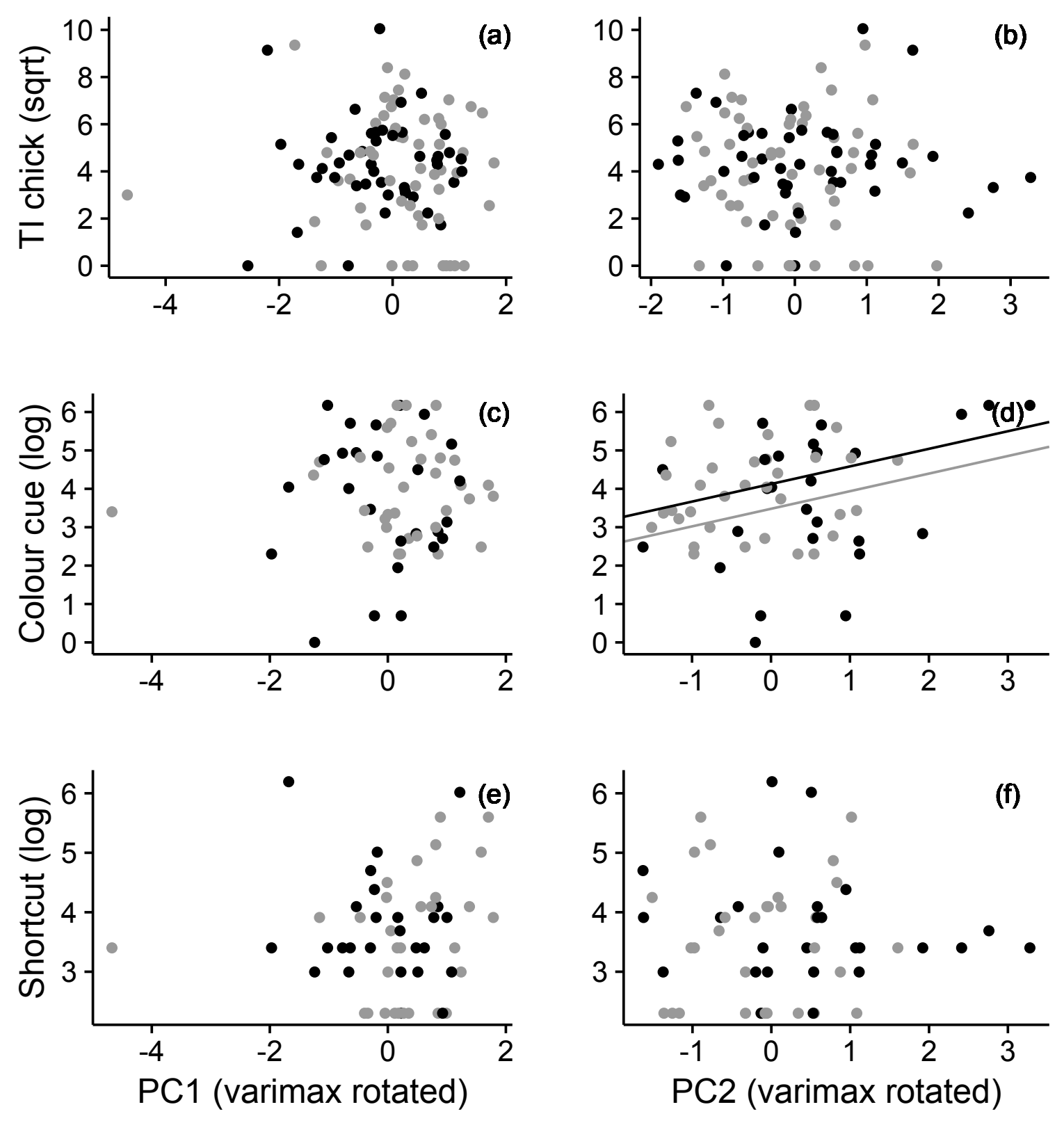


